\title{
A statistical approach to latitude measurements: Ptolemy's and Riccioli's geographical works as case studies
}

\author{
Luca Santoro \\ Via F. Verrengia 2, 84132 Salerno, Italy \\ Correspondence to: Santoro Luca (luca.scontr@gmail.com)
}

Received: 11 January 2017 - Revised: 28 April 2017 - Accepted: 4 July 2017 - Published: 7 August 2017

\begin{abstract}
The aim of this work is to analyze latitude measurements typically used in historical geographical works through a statistical approach. We use two sets of different age as case studies: Ptolemy's Geography and Riccioli's work on geography. A statistical approach to historical latitude and longitude databases can reveal systematic errors in geographical georeferencing processes. On the other hand, once exploiting the right statistical analysis, this approach can also lead to new information about ancient city locations.
\end{abstract}

\section{Introduction}

Historical methods underlying geographical coordinate measurements represent an interesting scheme in order to analyze the evolution, technical issues and mathematical theory that inspire modern geography. Much attention has to be given to longitude measurements, since these measurements introduce a major distortion source for geographical maps. Moreover, the longitude concept derives from the concurrence of observations and measurement procedures with respect to a reference place, so it can be considered a measure of time. For this purpose, Hipparchus considered Moon eclipse observations, and in the modern era, after Galileo, astronomers and geographers also used Jupiter moon eclipses. The interesting idea to measure a geographical quantity with time led to many philosophical considerations. In the past it was not generally used but replaced with estimates of distances between cities or places of interest. Such different schemes implied inhomogeneous error sources; in fact, time was measured only where it was possible to compute with Sun and water or sand clock; otherwise lengths were measured, with errors related to an undefined precision in direction and distance. Geographical coordinates were affected by many corrections during history, starting with Al-Khwarizmi's longitude corrections of Ptolemy's Geography in the Mediterranean area.
The latitude coordinate has not received the same attention, since magnitude variations have been related to measurement errors. To achieve such a coordinate measure one needs to define the angle between the zenith and Sun position at the equinox and, since this quantity does not change in time, it is very simple to obtain the right latitude estimate. The direct method used is to measure the gnomon shadow length at the equinox. An indirect approach was to measure the length at the solstice and, therefore, to measure the gnomon shadow length at the solstice. If we think about angle position and we denote the latitude with $\Phi$, the ecliptic with $\varepsilon$ and the angle of the Sun's position at summer solstice with $\beta$, it is simple to write the relation:

$\Phi=\beta+\varepsilon$.

We have to remember that the obliquity changes with time, as explained in Laskar (1986a, b), between a maximum of $24.5^{\circ}$ and a minimum of $22.04^{\circ}$ on a cycle of around 41000 years. From the beginning of human history, the obliquity has decreased, and since ancient times many astronomers have measured the ecliptic, e.g., Wittman (1979). In a different way, $\beta$ also changes its magnitude in time, but its value increases in a way that is coherent with respect to $\varepsilon$. Many studies on megalithic monuments (Thom, 1967, 1971, 1972, 1973, 1974, 1984; Thom et al., 1974) show an ecliptic at the solstice or equinox point (sunset or sunrise and moonset or moonrise), estimated in agreement with the respective 
epoch, although these results are still controversial; see Ruggles (1999).

The latitudes have to remain the same from the time of its definition, with the only change due to measurement errors. In this paper we study latitude changes between different epochs. We propose a statistical approach to study historical latitude measurements in order to understand if there are some systematic errors in the historic measurements and if it is possible to develop correlations with other physical measurements such as longitude.

\section{The method}

In the present investigation we will study the specific statistical distribution

$\Delta \Phi=\Phi_{\mathrm{an}}-\Phi_{\mathrm{t}}$

where $\Phi_{\mathrm{an}}$ is ancient latitude and $\Phi_{\mathrm{t}}$ is today's latitude for the same geographical location. A statistical analysis of the latitude deviation can allow us to investigate possible latitude position distortion, offering a new instrument to investigate historical geography and locate ancient cities.

During history, the accuracy of latitude measurements became more precise because of the improvement of methods and technologies. As a matter of fact, the most precise measurement of such a quantity is its last estimate, that is today's latitude. If measurements obtained during the same epoch are considered, one has a coherent set of latitudes measured with the same method and suitable error bars due to a random measurement process. The error theory is straightforward: if we have a coherent set with random error bar values, the set has to show a distribution with a random component (Gaussian, Laplace or logistic) with a mean peak. Thus, evaluating the distribution for historical measurement sets, one can evaluate whether errors are statistically random or if there are systematic problems.

In order to build a suitable latitude statistical distribution, the steps we have to follow are as follows: (1) we choose a historical latitudes set; (2) we identify the corresponding localities nowadays; (3) we calculate the latitude difference for the same geographic point and (4) we analyze the statistical distribution.

The first interesting point of this method is that it is necessary to identify the city, villages or geographical point of the historical map.

The case studies we will present in the next sections are the latitude sets encoded within Ptolemy's Geography (second century BC) and Tabula Latitudinum et Longitudinum of Giambattista Riccioli (1689). We have chosen these two latitude sets for specific reasons. Ptolemy's Geography is the oldest treaty on geography, summarizing almost all ancient knowledge about geography and measurement methods. All newer survey methods have to deal with this treaty, one of the most important in history. Riccioli's work, on the other hand, is a modern data set based on the first geodesy studies, developed soon after Tycho Brahe's epoch. At the end of the seventeenth century, geodesy's epoch begins; this new georeferencing scheme introduced a revolution for cartography. Furthermore, Riccioli in his work Astronomia Reformata, summarizes the bulk of ancient geographic studies in a critical way. He also analyzes all ecliptic measurements from Hipparchus' time, showing a deep knowledge of problems related to measures, astronomy and geography. Riccioli's work was done at a time in which the ecliptic value is very close to the real estimate, so that the distribution peak of latitude differences between Riccioli's estimates and the modern calculations is expected to be close to zero.

\section{Ptolemy's set}

Ptolemy's work had a great impact on knowledge for astronomy and geography until Copernicus' revolution. The books Almagest (Mathematiké sýntaxis) and Geography (Geōgraphikè hyphègēsis) represent a benchmark in this sense. The Almagest is one of the most studied ancient treaties, but it still raises issues without conclusive answers. Delambre $(1817,1819)$ already remarked on the problem of data used by Ptolemy. This triggered research on Ptolemy's sources and how he compiled the treaty, inheriting and summarizing works by Eratosthenes, Hipparchus, Marinus of Tyre, Pytheas, Eudoxus of Cnidus and Posidonius. Newton (1973, 1974, 1977), Britton (1969) and, more recently, Grasshoff (1990), Jones (2002) and Shcheglov (2006, 20032007) also address these topics. One idea is clear: Ptolemy used data from a different epoch, partly measured by himself, but he assumed the same ecliptic from predecessors (maybe Hipparchus or Eratosthenes measured $23^{\circ} 51^{\prime} 20^{\prime \prime}$ ), despite some star observations showing a different ecliptic value, $23^{\circ} 43^{\prime} 06^{\prime \prime}$ which is more accurate in comparison to the epoch ecliptic magnitude.

The Geography treaty has also been widely studied in time: the origin of projections has been analyzed as well as the manuscripts' dissemination and a significant investigation has been pursued about the positioning of ancient lost cities. Starting from the translation of theoretical books, e.g., Berggen and Jones (2000), a complete coordinates database has been published in Stuckelberg and Grasshoff (2006), while Isaksen (2011) provided a general discussion on the accuracy of coordinates in the Geography; on the other hand, some insights on the geostatistical methods are provided in Livieratos et al. (2008) and Tsorlini (2009a, b), and finally Marx (2011) developed a more mathematical analysis concerning statistical methods.

A significant aim is to estimate the coordinates' error bars, in order to understand the position of lost cities. Nowadays we know that the longitude coordinate has the greater error bar but few works have been published on numerical methods to rectify the longitude coordinate, showing the 


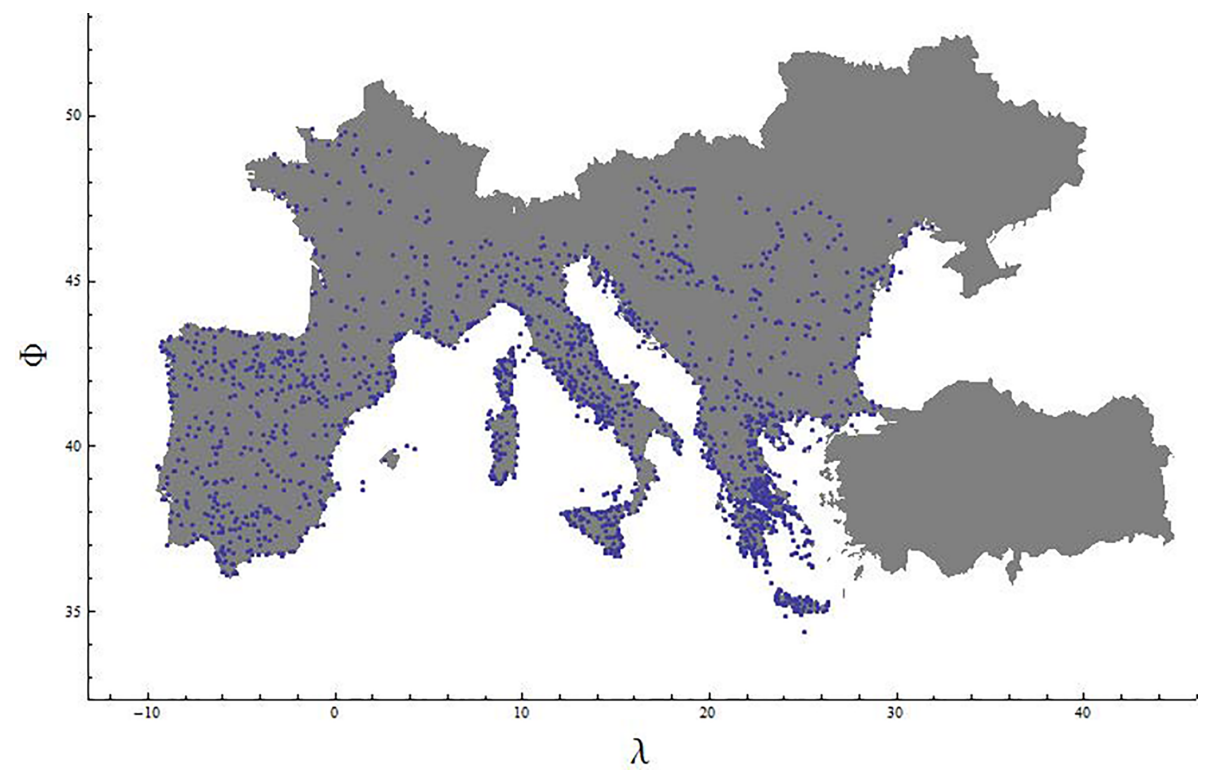

Figure 1. Map of contemporary counterparts of Ptolemy's locations considered in the present analysis on a map (blue dots).

longitude increase from west to east, as in Marx (2012a) and Russo (2013). Based on this idea, a regional catalogue has been published, that considers some parts of Ptolemy's oikumene, with Ptolemy's coordinates and contemporary counterpart locations (see Kleineberg et al., 2010, 2012, and in Marx and Kleineberg, 2012). The methodology used in the Geography is the same as in the Almagest, as Grasshoff (2014) argued. A general feature of Ptolemy's works is to be heterogeneous; this was highlighted for the $\mathrm{Al}$ magest and it is accepted for the Geography too. There is no unique polynomial relation to calculate the modern location from the historic coordinates over all oikumene, as discussed in Marx (2012b) but only regional relations.

We know very well that Ptolemy used travel itineraries, circumnavigations and other regional data from ancient chorography probably preserved in Alexandria's library. It is still an open issue of how Ptolemy built geographic maps and coordinates from a non astronomical data set. Grasshoff (2014) argued that coordinates of entire maps are not built from astronomical data but only from knowledge of distances between locations.

In the present work we focus on investigating latitude measurements, as they are not affected by the value assumed for the Earth's radius (as longitude measurements would be). Great distortions have been found for certain parts of Ptolemy's oikumene, as shown in Marx (2014). A suitable approach is to only consider the Mediterranean zone, which was better known because of the presence of the Roman Empire. We use the works of Kleineberg et al. (2012) and, Marx and Kleineberg (2012) in order to draw Ptolemy's geographic locations distribution and the contemporary corrected counterparts. The distribution of locations is shown in Fig. 1.

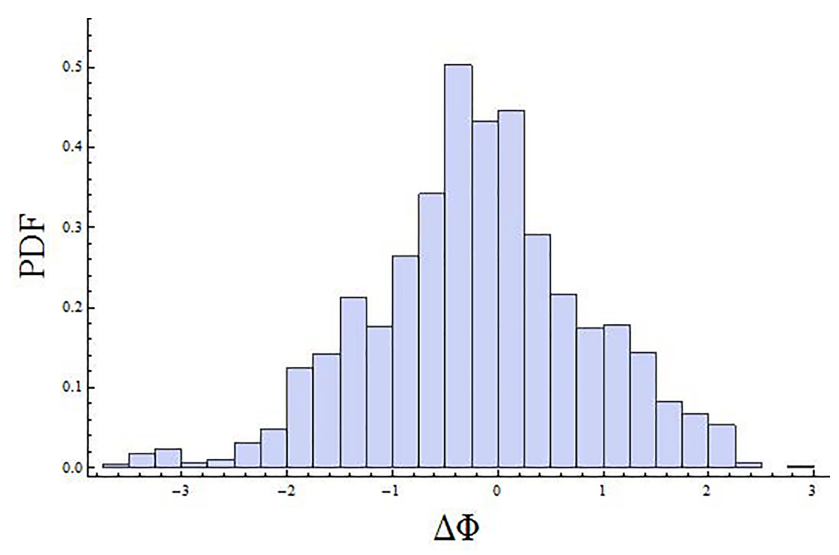

Figure 2. Histogram of latitude deviation with bin width of $15^{\prime}$ for Ptolemy's location set.

The number of cities in this catalogue is 1885 , so the statistical set is quite large, although it is lacking North Africa and the Middle East. We extract the latitude difference as in Eq. (2) for the set of cities collected, where $\Phi_{a n}$ is Ptolemy's latitude (ancient) and $\Phi_{\mathrm{t}}$ is today's latitude for the same geographical location. Figure 2 shows a histogram of the empirical PDF (probability density function) of a statistical quantity corresponding to the number of objects with same $\Delta \Phi$.

The histogram represents the empiric distribution of the latitude difference. We can see the structure of the distribution is well defined and quite symmetric but not centered at 0 . We know Ptolemy's Geography had different, inhomogeneous sources and data came from astronomical, itinerary and periploi sources. If we accept this idea, we need to be sure that inhomogeneous data are better described by mixing 


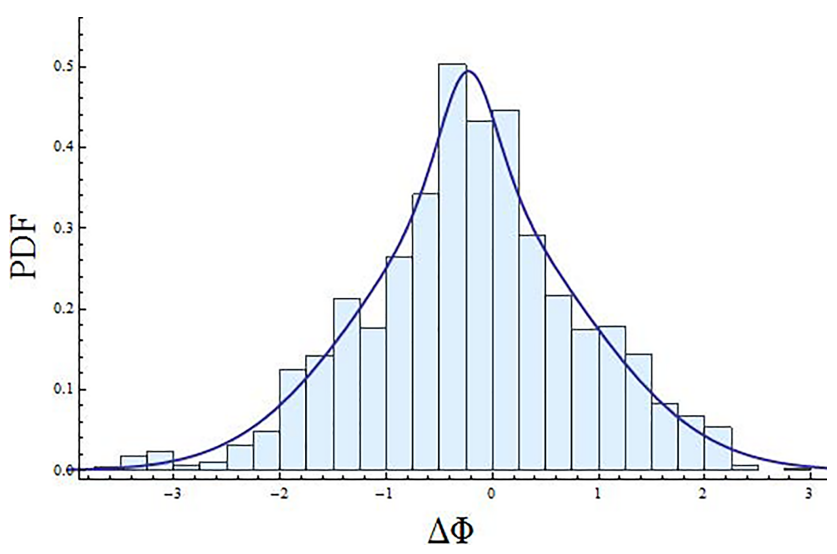

Figure 3. Histogram with bin width of $15^{\prime}$; the blue continuous curve is the statistical distribution $f$ inferred for Ptolemy's set.

statistical distributions. For this purpose, we try three different statistical distributions for a random effect and their linear combination: Gaussian, Laplace and logistic distributions and also with a linear combination. We use the maximum likelihood method, and we have to reject Gaussian, Laplace and logistic distributions taken singly because the Cramér-von Mises statistic test is negative (statistic test for goodness of distribution). We have to reject the combinations Gaussian-Laplace and Laplace-logistic for the same reason. We have obtained best results assuming a distribution

$f=\alpha \cdot f_{\mathrm{G}}+(1-\alpha) \cdot f_{\mathrm{Lo}}$,

where $f_{\mathrm{G}}$ is the Gaussian statistical distribution, $f_{\mathrm{Lo}}$ is the logistic one and $\alpha$ is the width of each distribution in the mixing. The results are summarized in Fig. 3 and Table 1 .

The fundamental result is that there is a systematic error in latitude. Such an effect is suggested by a non-zero statistical distribution mean of the latitude errors; it corresponds to $12^{\prime}$ and it is not negligible due to a large sample size. We can conclude that overall in the Mediterranean area the latitude is underestimated.

The question now is how we can explain this distortion. The possible sources of latitude error are many. We can only point out some possible explanations in this exploratory analysis focusing on the Mediterranean area. First of all, we check whether a correlation between longitude and latitude measurements exists. In principle, longitude/latitude measurements are uncorrelated to one another. However, almost all ancient latitude values are not correctly measured but derived from the shadow length using a gnomon in the sunlight during two particular days: summer solstice and equinox. This procedure was also used to find the geographic south and north positions, crucial for defining longitude. But obviously this geometric procedure is affected by a measurement error, and consequently it may thus spread to both latitude and longitude measurements. Within the considered sample there are certainly a lot of astronomical data sets forming
Table 1. Statistical analysis and tests for $f$ statistical distributions for Ptolemy's set: $\mu$ is the mean, $\sigma$ is the standard deviation, RMSE is the root mean square error, Cramér-von Mises $P$ value is the goodness parameter and $\alpha$ is the mixing parameter.

\begin{tabular}{lr}
\hline$\mu$ & -0.1987 \\
$\sigma$ & 0.9945 \\
RMSE & 0.069 \\
Cramér-von Mises $P$ value & 0.65854 \\
$\alpha$ & 0.859 \\
\hline
\end{tabular}

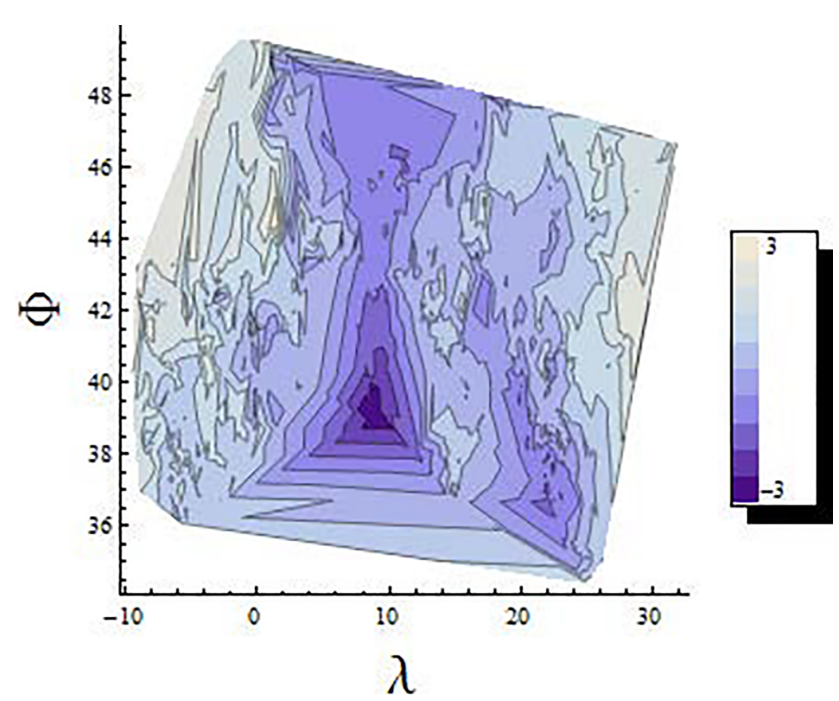

Figure 4. Latitude difference plot for each location set. The axes are contemporary latitude and longitude.

a first step to create a reticulum of parallels and meridians. As a second step, additional data coming from itineraries or knowing distances in general are added to this first set of data. In such a case, a lower level of accuracy characterizes latitude and longitude estimations. Different source data are affected by different error bars, and longitude estimations reveal a strong possibility of correlation with latitude.

In Fig. 4 we plot a counterplot to show and investigate possible latitude-longitude correlations. It is created starting from cities' geographical coordinates and their own $\Delta \Phi$. So areas with the same $\Delta \Phi$ are mapped and visualized in the latitude-longitude plot.

In Fig. 5, we show an analogous plot for longitude. As we can see in Figs. 4 and 5, the counterplots are very useful to observe how $\Delta \lambda$ and $\Delta \Phi$ are mapped and spatially distributed. We do not consider the longitude offset; it exists but it does not change the counterplot. In the latitude counterplot we can see a sort of butterfly correlation, where the spatial distribution of $\Delta \Phi$ is not random, and in the longitude counterplot $\Delta \lambda$ has a gradient from west to east, but this is well known and used to search for the modern location counterpart. In the area where the $\Delta \lambda$ gradient is highest, i.e., where $\Delta \lambda$ changes fast with longitude (around longitude 


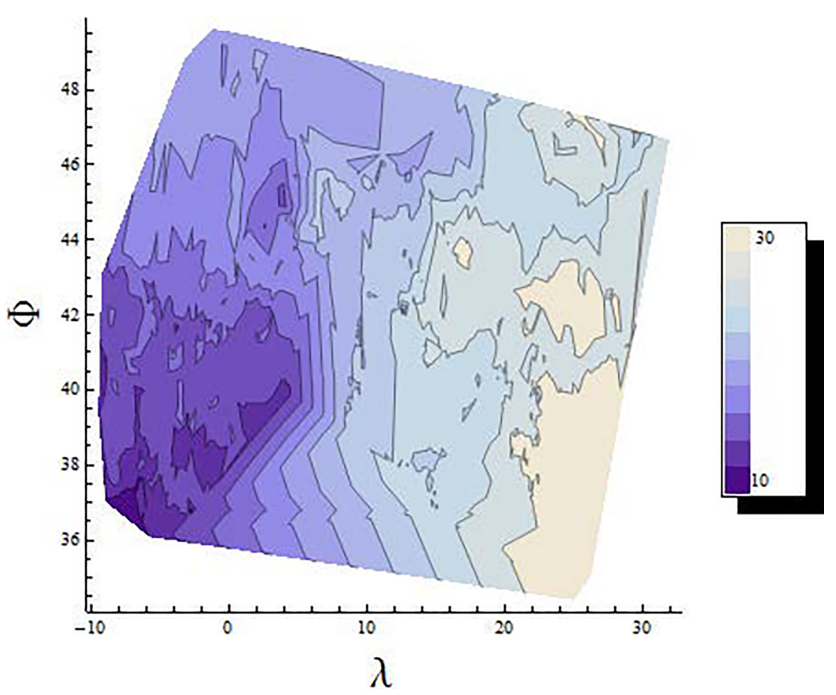

Figure 5. Longitude difference plot for each location set. The axes are contemporary latitude and longitude.

$\left.10^{\circ}\right), \Delta \Phi$ is negative and lowest. Where $\Delta \lambda$ is quite constant (side areas), $\Delta \Phi$ is positive. There seems to be a general correlation between latitude and longitude and consequently a correlation between latitude and longitude error bars, possibly owing to the dependence of both on knowledge of the meridian. Thinking of rulers used for the measurements, it is realistic to think that most latitude measurements have had $10^{\prime}$ as error bars. If errors in latitude/longitude propagate to one another, and there is the same precision, we have roughly a typical error of measurement. This can explain at least part of the statistical spread. From a general point of view, we can hypothesize a general error propagation like

err $=a \Delta \Phi+b \Delta \lambda$ with $a b \neq 1$,

but the difficulty is how we can find the parameters $a$ and $b$ if we are not able to find the error bars for latitude and longitude in a separate way.

Another possible source for latitude distortion comes from the definition in Eq. (1). Ptolemy collected many data using different types of sources and methods, produced in different years: we know he used latitude and longitude estimates from Babylonian tradition too. Using the gnomon shadow length at the summer solstice, he estimated the latitude making use of an ecliptic estimate, maybe by himself or from ancient astronomers like Hipparchus and Eratosthenes. In this case it is possible he did not adopt $\beta$ and $\varepsilon$ in a coherent way but from various time periods. Consequently the resulting latitude estimation is underestimated or overestimated, since the data turn out to be affected by the ecliptic change.

The question now is how we can distinguish all the biasing contributions. A feasible procedure is to individuate and investigate regional zones where latitude and longitude estimations are correlated in a coherent way. The motivation for this is plausible because there are some coherent areas, that

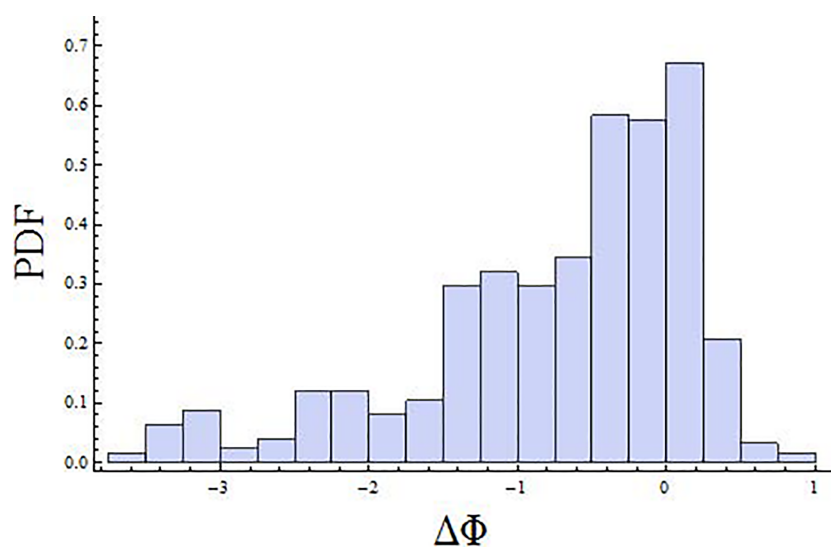

Figure 6. Histogram with bin width of $15^{\prime}$ for mainland Italy, Sicily and Sardinia.

is astronomical estimates, and other estimates around central cities came from itinerary distances for each geographical area. As an example, we plot $\Delta \Phi$ for mainland Italy, Sicily and Sardinia in Fig. 6.

This histogram plot shows a very different systematic error with respect to the overall biasing effect obtained in Fig. 2. We can conclude that the distortion sources for this region must be different. From this example it is easy to understand that a better comprehension of errors in historic coordinate sets needs further studies taking into account the statistical data as well as historical sources. The regional methods proposed by Grasshoff (2014) seem a highly promising approach.

\section{Riccioli's set}

Riccioli's works, during the seventeenth century, are famous because they focus on the inaccuracy of Copernicus' heliocentric system. He is in favor of a geocentric model, and each work tends to demonstrate it. Delambre (1821) judges Riccioli's works to be not very useful to modern astronomy. The Jesuit Riccioli lived in a century dominated by the revolutionary figures Galileo and Copernicus, and the Church tried to stop their ideas. Beyond the theological and philosophical discourse, as shown in Graney (2012), Riccioli had the fame to summarize every knowledge about astronomy, physics and geography. He discussed measurement methodology in his works, mostly in Almagestum novum and Astronomia reformata. In the latter, he analyzed the ecliptic measure from Hipparchus and Ptolemy, which is interesting for our work. He concluded that the correct value for the ecliptic is $23^{\circ} 30^{\prime}$ and that the ancient astronomers were wrong, adding, for theological reasons, the notion that the ecliptic is constant. $\mathrm{He}$ made many astronomical observations with Grimaldi and, more interesting for our purpose, measured the meridian (Hoefer, 1873). In his works Geographice et hydrographice reformatce libri duodecim and Tabula latitudinum et longi- 


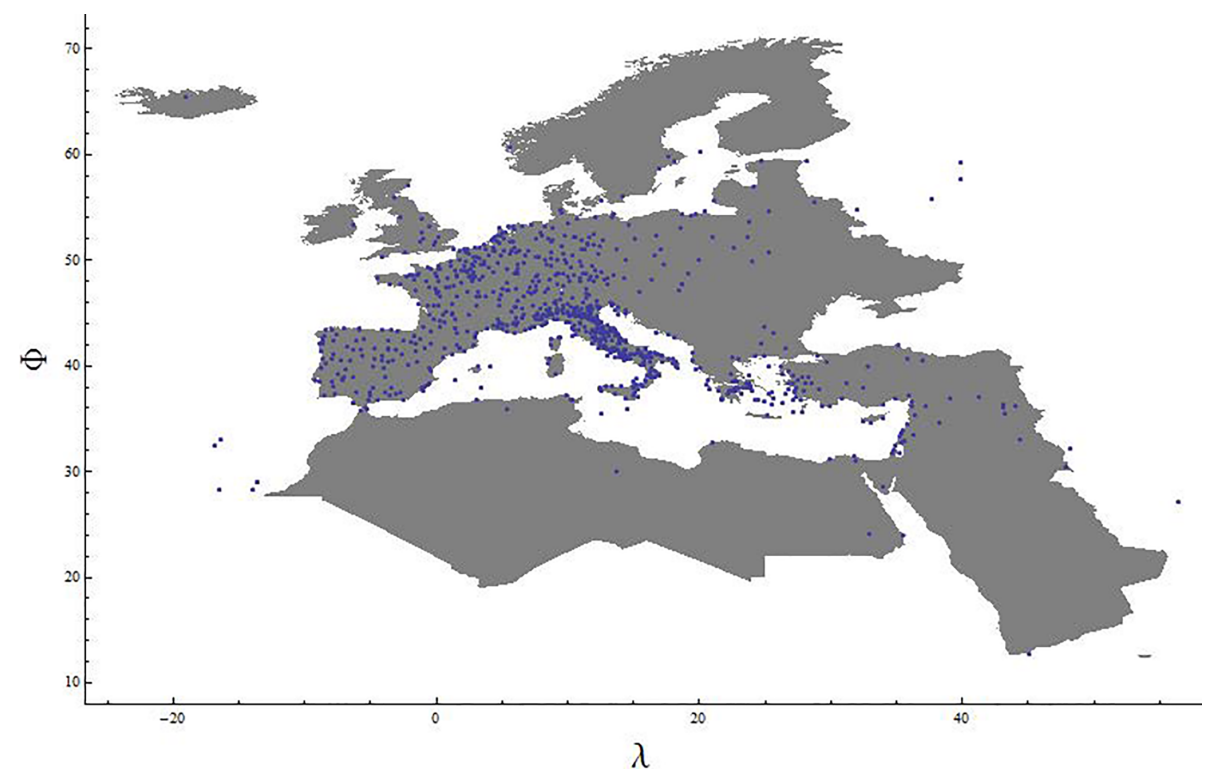

Figure 7. Map of contemporary counterparts (blue dots) of Riccioli's locations considered in the present analysis.

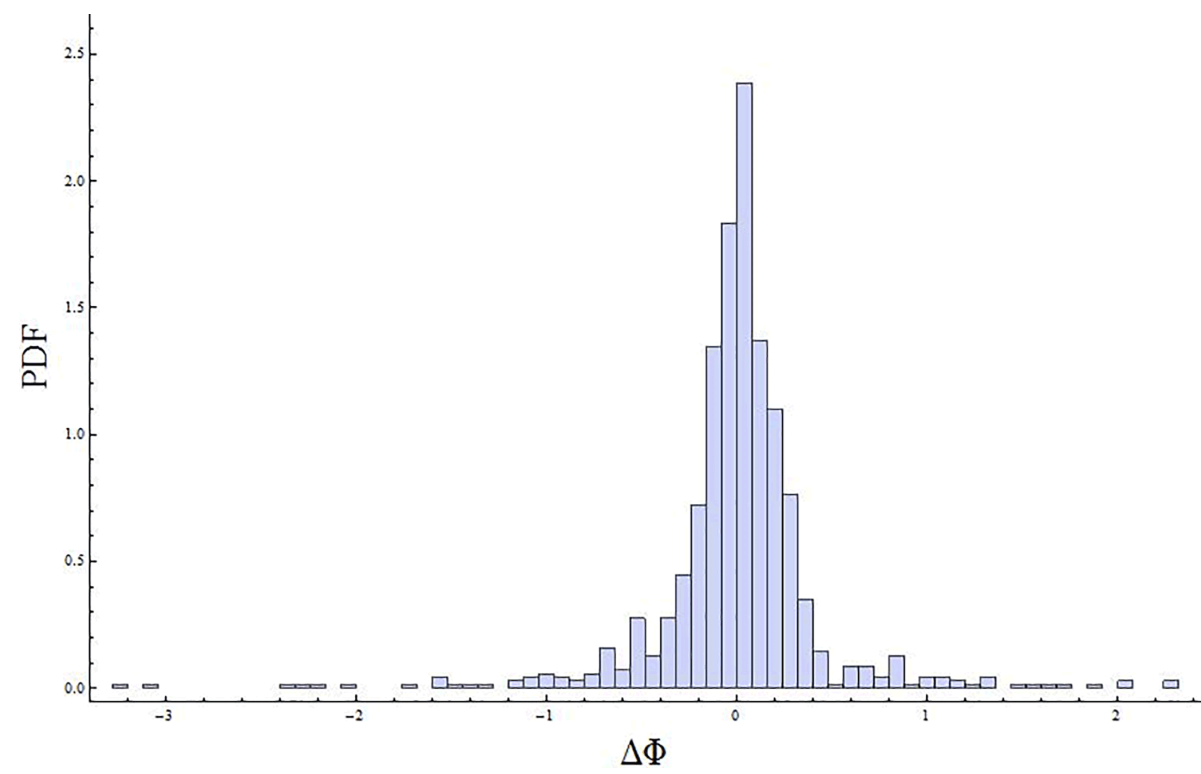

Figure 8. Histogram with bin width of $5^{\prime}$ for Riccioli's set of latitudes.

tudinum, he collected and corrected many geographical measurements. His work on geography remained a reference point for next-generation cartographers like Rizzi Zannoni and Fontana, as in Memorie di Matematica (1826). Unlike astronomy, geography was not debated on a theological level, so Riccioli's geographical works are trustworthy. For these reasons we chose Tabula latitudinum et longitudinum to analyze with our method. To build a homogeneous latitude set, we have to chose the same geographical zone as we did for Ptolemy's Geography in the previous section. The geographical location distribution is shown in Fig. 7.
The contemporary counterparts are easier to recognize because, from the seventeenth century, many locations have kept the same name and most cities existed already. The histogram set of latitude differences for Riccioli, analogous to Fig. 2 for the Ptolemy case, is shown in Fig. 8.

We limited the statistical analysis to the $[-0.5,0.5]$ interval because we had some outliers that, however, do not significantly change the statistical results. The $\Delta \Phi$ histogram structure is quite symmetric around 0 and well defined. We redo the same statistical analysis as for the Ptolemy set in the previous section. We use the maximum likelihood method, 
Table 2. Statistical analysis and tests for $f$ statistical distributions for the Riccioli set: $\mu$ is the mean, $\sigma$ is the standard deviation, RMSE is the root mean square error, Cramér-von Mises $P$ value is the goodness parameter and $\alpha$ is the mixing parameter.

\begin{tabular}{lr}
\hline$\mu$ & -0.003 \\
$\sigma$ & 0.12 \\
RMSE & 0.016 \\
Cramér-von Mises $P$ value & 0.511 \\
$\alpha$ & 0.248
\end{tabular}

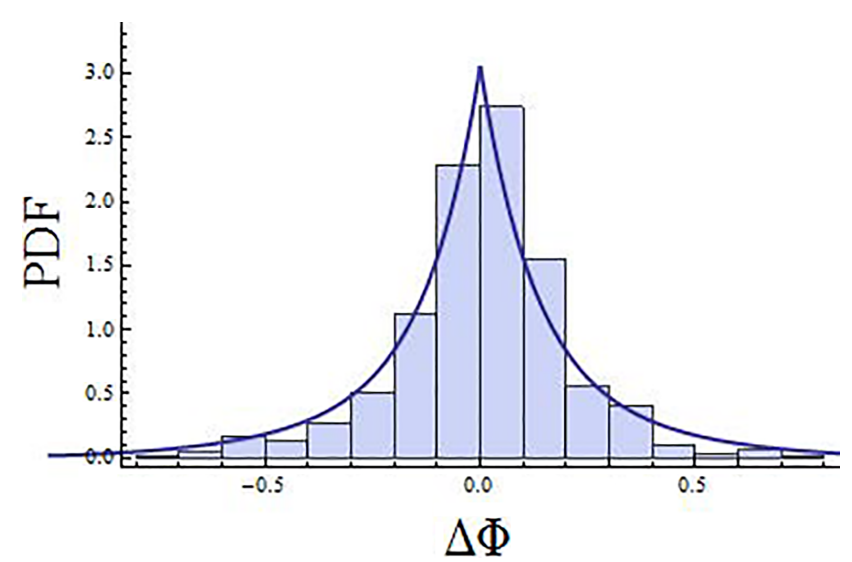

Figure 9. Histogram with bin width of $10^{\prime}$; the blue continuous curve is the statistical distribution inferred for Riccioli's set.

and we have to reject Gaussian, Laplace and logistic distributions taken singly because the Cramér-von Mises statistic test is negative, as in the Ptolemy case. We have to reject the combinations Gaussian-logistic and Laplace-logistic for the same reason. The best result in this case is

$f=\alpha \cdot f_{\mathrm{G}}+(1-\alpha) \cdot f_{\mathrm{L}}$,

where $f_{\mathrm{G}}$ is the Gaussian statistical distribution, $f_{\mathrm{L}}$ is the Laplace distribution and $\alpha$ is the weight of each distribution in the mixing. We found the $\alpha$ parameter through the maximum likelihood method. The results are given in Fig. 9 and Table 2.

As we can see from the results, the best fit is with a composite distribution in which $\mu$ is very near to 0 and the standard deviation $\sigma$ is around $7^{\prime}$. During Riccioli's time, many observational instruments had a better accuracy than in Ptolemy's time and is reflected in a standard deviation $\sigma$ smaller than in Ptolemy's case. The existence of outliers and the fit with a linear condition of distributions suggest there are irregularities in the geographical data, but in Riccioli's case it is quite mild. From statistical analysis, no systematic error or distortion is evident. We can exclude all types of correlations between latitude and longitude measurements, because $\Delta \Phi$ is a spatially random distribution. This can be easily observed from the counterplot for latitude and longitude in Figs. 10 and 11.

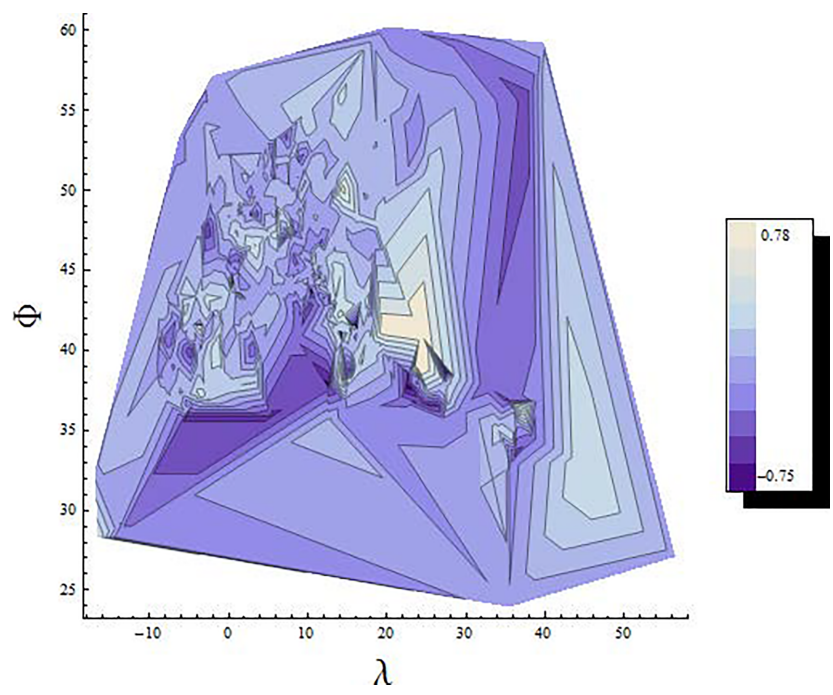

Figure 10. Latitude difference plot for each location set. The axes are contemporary latitude and longitude.

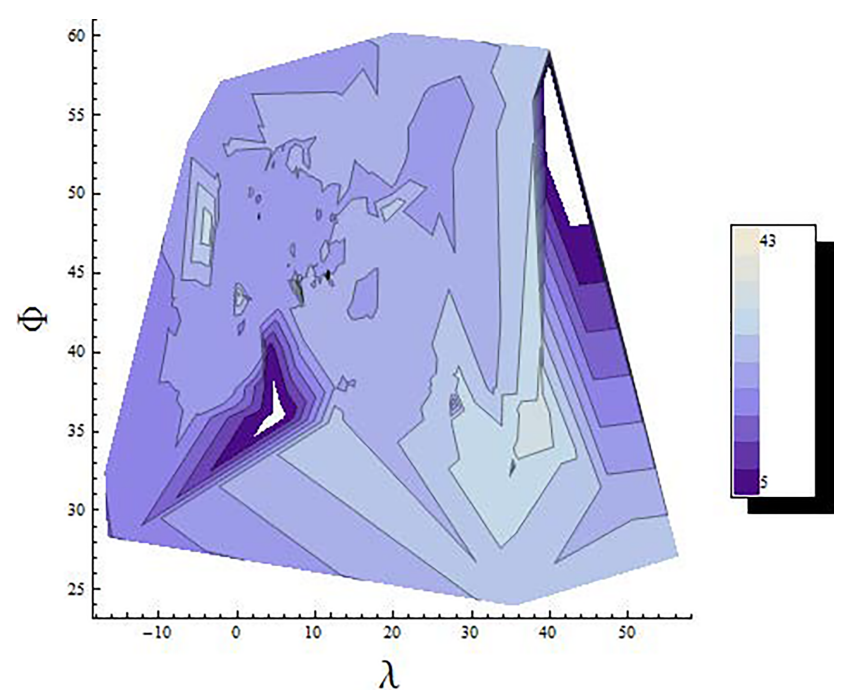

Figure 11. Longitude difference plot for each location set. The axes are contemporary latitude and longitude.

The main result is that there is no significant systematic error or any latitude-longitude correlation in the entire geographical area. Riccioli's set has coherent astronomical data and latitude estimates. The $\Delta \Phi$ study shows quite a random distribution due to a simple procedure to measure latitude, as we can expect from any measurement.

\section{Conclusions}

In order to thoroughly understand how geographic locations given in historic works were measured or calculated, studies on the statistics of location values are a promising method. In the present exploratory work, we highlight the relevance of 
the statistics of latitude measurements. In particular, we focus on the statistical distribution of $\Delta \Phi$ as tracer to analyze systematic errors, distortions, latitude-longitude correlations and measurement uncertainties. We discuss the statistical results of two case studies: Ptolemy's and Riccioli's geographical works. As shown, the statistical distribution of $\Delta \Phi$ for Ptolemy shows properties (peak position and standard deviation) best explained with an inhomogeneity in measurement, data collection, methods and measurement precisions. For future works, studies like ours should be brought together with studies on longitude and historical sources; this could bring about insights on the circulation of data and methods among ancient astronomers and scientists. In Ptolemy's case, it is curious that the longitude gradient maximum zone is very near to the Carthage longitude, one of the locations of ancient lunar eclipse observations probably used by Ptolemy. Statistical analysis like ours, but dealing with other classical works, may show further correlations between latitude and longitude measurements and give us more information about ancient city locations.

Data availability. The author collected the latitude and longitude data to built Ptolemy's and Riccioli's set. For Ptolemy's set the author used the data contained in Marx and Kleineberg (2012) and in Kleineberg et al. (2012). For Riccioli's set the author used the data contained in Ricciolo (1689).

Competing interests. The author declares that they have no conflict of interest.

Acknowledgements. I would like to thank A. Pagliano and S. Capozziello for their continued support, S. Hachinger and A. Troisi for friendship, and last but not least the referees and the handling editor, S. Papamarinopoulos, for the good suggestions.

Edited by: Stavros Papamarinopoulos

Reviewed by: two anonymous referees

\section{References}

Berggren, J. L. and Jones, A.: Ptolemy's Geography, An Annotated Translation of the Theoretical Chapters, Princeton University Press, Princeton, USA, 2000.

Britton, J. P.: Ptolemy's Determination of the Obliquity of the Ecliptic, Centaurus, 14, 29-41, 1969.

Delambre, J.-B.: Histoire de l'Astronomie Ancienne, Courcier, Imprimeur Libraire pour les Sciences, Paris, 1817.

Delambre, J.-B.: Histoire de l'Astronomie du Moyen Age, Courcier, Imprimeur Libraire pour les Sciences, Paris, 1819.

Delambre, J.-B.: Histoire de l'Astronomie moderne, Paris, vol. I, 679 pp., 1821.
Graney, C. M.: 126 Arguments Concerning the Motion of the Earth, as Presented by Giovanni Battista Riccioli in his 1651 Almagestum Novum, J. Hist. Astron., 43, 215-226, 2012.

Grasshoff, G.: The history of Ptolemy's star catalogue, SpringerVerlag, New York, 1990.

Grasshoff G.: Ptolemy and Empirical Data, in: Astroculture. Figurations of Cosmology in Media and Arts, edited by: Neef, S., Sussmann, H., and Boschung, D., Wilhelm Fink Verlag, München, 2014.

Hoefer, F.: Histoire de l'astronomie, Libraire Hachette, Paris, 1873.

Isaksen, L.: Lines, damned lines and statistics: unearthing structur in Ptolemy's Geographia, e-Perimetron, 6, 254-260, 2011.

Jones, A.: Eratosthenes, Hipparchus, and the obliquity of the ecliptic, J. Hist. Astron., 33, 15-19, 2002.

Kleineberg, A., Marx, C., Knobloch, E., and Lelgemann, D.: Germania und die Insel Thule. Die Entschlüsselung von Ptolemaios' "Atlas der Oikumene", Wissenschaftliche Buchgesellschaft, Darmstadt, 2010.

Kleineberg, A., Marx, C., and Lelgemann, D.: Europa in der Geographie des Ptolemaios. Die Entschlüsselung des "Atlas der Oikumene": Zwischen Orkney, Gibraltar und den Dinariden, Wissenschaftliche Buchgesellschaft, Darmstadt, 2012.

Laskar, J.: Secular Terms of Classical Planetary Theories Using the Results of General Relativity, Astron. Astrophys., 157, 59-70, 1986a.

Laskar, J.: Erratum: Secular terms of classical planetary theories using the results of general theory, Astron. Astrophys., 164, 437, $1986 b$.

Livieratos, E., Tsorlini, A., Boutoura, C., and Manoledakis, M.: Ptolemy's Geographia in digits, e-Perimetron, 3, 22-39, 2008.

Marx, C.: On the precision of Ptolemy's geographic coordinates in his Geographike Hyphegesis, Hist. Geo Space. Sci., 2, 29-37, https://doi.org/10.5194/hgss-2-29-2011, 2011.

Marx, C.: Rectification of the ancient geographic coordinates in Ptolemy's Geographike Hyphegesis, Hist. Geo Space. Sci., 3, 99-112, https://doi.org/10.5194/hgss-3-99-2012, 2012a.

Marx, C.: Investigation of the coordinates in Ptolemy's Geographike Hyphegesis Book 8, Arch. Hist. Exact Sci., 66, 531555, 2012 b.

Marx, C.: Rectification of position data of Scotland in Ptolemy's Geographike Hyphegesis, Surv. Rev., 46, 231-244, 2014.

Marx, C. and Kleineberg, A.: Die Geographie des Ptolemaios. Geographike Hyphegesis Buch 3: Europa zwischen Newa, Don und Mittelmeer, epubli GmbH, Berlin, 2012.

Memorie di Matematiche e di Fisica della società italiana delle Scienze residente in Modena: vol. 20, Camerale, Modena, p. 110, 1826.

Newton, R. R.: The Authenticity of Ptolemy's Parallax Data - Part I, Q. J. Roy. Astron. Soc., 14, 367-388, 1973.

Newton, R. R.: The Obliquity of Two Millenia Ago, Mont. Notic. Roy. Astron. Soc., 169, 331-342, 1974.

Newton, R. R.: The crime of Claudius Ptolemy, J. Hopkins University Press, Baltimore, London, 1977.

Ricciolo, G.: Tabula Latitudinum et Longitudinum, Susannam Christinam, Matthaei Cosme rovij, Vienna, 1689.

Ruggles, C.: Astronomy in Prehistoric Britain and Ireland, Yale University Press, New Haven, London, 1999. 
Russo, L.: Ptolemy's longitude and Eratosthenes' measurement of the Earth's circumference, Math. Mech. Complex Syst., 1, 6779, 2013.

Shcheglov, D.: Eratosthenes' Parallel of Rhodes and History of the System of Climata, Klio, 88, 351-359, 2006.

Shcheglov, D.: Hipparcus' Table of Climata and Ptolemy's Geography, Orbis Terrarum, Amsterdam, 159-191, 2003-2007.

Stuckelberg, A. and Grasshoff, G.: Ptolemaios Handbuch der Geographie, Schwabe, Basel, 2006.

Thom, A.: Megalithic sites in Britain, Oxford University Press, Oxford, 1967.

Thom, A.: The astronomical significance of the large Carnac menhirs, J. Hist. Astron., 2, 147-160, https://doi.org/10.1177/002182867100200301, 1971.

Thom, A.: The Carnac alignments, J. Hist. Astron., 3, 11-26, https://doi.org/10.1177/002182867200300103, 1972.
Thom, A.: The Kerlescan cromlechs, J. Hist. Astron., 4, 169-173, https://doi.org/10.1177/002182867300400303, 1973.

Thom, A.: The Kermario alignments, J. Hist. Astron., 5, 30-47, https://doi.org/10.1177/002182867400500104, 1974.

Thom, A.: The two major Megalithic observatories in Scotland, J. Hist. Astron., 15, 129-148, 1984.

Thom, A., Stevenson Thom, A., and Strang Thom, A.: Stonehenge, J. Hist. Astron., 5, 71-90, https://doi.org/10.1177/002182867400500201, 1974.

Tsorlini, A.: Higher order systematic effect in Ptolemy's Geographia coordinate description of Iberia, e-Perimetron, 4, 117130, 2009a.

Tsorlini, A.: Spatial distribution of Ptolemy's Geographia coordinate differences in North Mediterranean eliminating systematic effets, e-Perimetron, 4, 247-266, $2009 \mathrm{~b}$.

Wittmann, A.: The Obliquity of the Ecliptic, Astron. Astrophys., 73, 129-131, 1979. 